\title{
Cognitive deficits in chronic pain patients, in a brief screening test, are independent of comorbidities and medication use
}

\author{
Déficits cognitivos em pacientes com dor crônica, em uma avaliação breve de triagem, são \\ independentes de comorbidades e uso de medicações \\ Karen dos Santos Ferreira ${ }^{1,2}$, Gabriela Zucatto Oliver², Débora Carinhato Thomaz², Caroliny Trevisan \\ Teixeira², Maria Paula Foss ${ }^{1}$
}

\begin{abstract}
Objective: To describe and analyze cognitive aspects in patients with chronic pain and a control group without pain. Method: A case-control study was conducted on 45 patients with chronic pain and on 45 control subjects. Data including pain diagnosis, comorbidities and medication used, were evaluated. Cognitive tests, such as the Montreal Cognitive Assessment (MoCA), Verbal Fluency Test, Clock Drawing Test and Stroop Test, were applied. Results: Patients with chronic pain showed a poorer performance, as shown by the scores of the MoCA test $(p<0.002)$, Verbal Fluency Test $(p<0.001)$, Clock Drawing Test $(p=0.022)$ and Stroop Test $(p<0.000)$. Chronic pain variable $(p=0.015$, linear regression model) was an independent factor for results obtained with the MoCA. Conclusion: Patients with chronic pain showed a poorer performance in a brief screening test for cognitive impairment not related to confounding variables, as comorbidities and pain-medication use.
\end{abstract}

Keywords: chronic pain; cognition; memory.

\section{RESUMO}

Objetivo: Descrever e analisar aspectos cognitivos em pacientes com dor crônica e um grupo controle sem dor. Método: Um estudo de caso-controle foi conduzido em 45 pacientes com dor crônica e 45 controles. Dados incluindo diagnóstico da dor, comorbidades e medicações utilizadas foram avaliados. Foram aplicados testes cognitivos, tais comoMontreal Cognitive Assessment, Teste da Fluência verbal, Teste do relógio e Teste de Stroop. Resultados: Pacientes com dor crônica apresentaram uma pior performance, em scores do MoCA $(p<0.002)$, Fluência verbal $(p<0.001)$, Teste do relógio $(p=0.022)$ e Stroop $(p<0.000)$. Dor crônica $(p=0.015$, modelo de regressão linear) foi um fator independente para os piores resultados obtidos no MoCA. Conclusão: Pacientes com dor crônica apresentaram uma pior performance em uma avaliação breve para comprometimento cognitivo, não relacionada a variáveis confundidoras, como comorbidades e medicações utilizadas para dor.

Palavras-chave: dor crônica; cognição; memória.

Chronic pain leads to physical and emotional stress in patients and caregivers, as well as high financial and social costs for the population ${ }^{1,2,3}$. The world prevalence of chronic pain ranges, in general, from 7 to $40 \%^{4}$. There is a predominance of affected women, aged 45 to 65 years ${ }^{4}$.

Patients with chronic pain frequently report cognitive complaints that impair social situations and daily life activities ${ }^{5,6,7}$. The exact mechanism underlying the complex relationship between pain, memory and attention is not fully understood, however it is known that the same neural networks used for many cognitive functions are also used for nociceptive functions. Clinical observation has revealed that many persons with chronic pain report loss of memory and concentration ${ }^{8,9}$.

The literature about chronic pain and cognition showed a small to moderate impairment in executive function performance ${ }^{10}$ and concluded that memory impairment frequently occurs in chronic pain patients but did not specify the type of memory impairment (working memory, long-term memory, autobiographical memory ${ }^{11}$. A previous study reported that

'Universidade de São Paulo, Faculdade de Medicina de Ribeirão Preto, Hospital Universitário, Ribeirão Preto SP, Brasil;

${ }^{2}$ Centro Universitário Barão de Mauá, Faculdade de Medicina, Ribeirão Preto SP, Brasil.

Correspondence: Karen dos Santos Ferreira; Divisão de Neurologia, Hospital Universitário, Faculdade de Medicina de Ribeirão Preto, Universidade de São Paulo;Avenida Bandeirantes, 3900;14048-900 Ribeirão Preto SP, Brasil; E-mail: karenferreira@usp.br

Conflict of interest: There is no conflict of interest to declare.

Received 19 August 2015; Received in final form 22 November 2015; Accepted 01 February 2016. 
healthy controls performed better in working memory tests, in comparison to patients with chronic pain, corroborating most investigations of cognition and chronic pain ${ }^{12}$. Only one study reported that these patients interpreted numbers differently from other people ${ }^{13}$.

Although all these studies reported cognitive impairment in chronic pain patients in comparison to normal controls, a consensus about their real dysfunctions was not reached. New insights to this matter could be provided by an expanded battery of tests with more specific domains and standardized methods. However, issues like the requirement of specialized professionals who may not be available in the primary care team, such as neuropsychologists, the length of time needed for the tests to be fully completed, and the lack of standardized testing methods previously validated to a given population are barriers to be considered.

It can also be seen that studies of patients with chronic pain have not taken into consideration many confounding variables such as medication used, and comorbidities such as depressive and anxiety symptoms. Some previous studies, have associated psychological aspects to problems in cognitive areas ${ }^{14}$ and others results have indicated that, independently of depressive symptoms, basic neurocognitive functions are impaired in patients with severe chronic pain ${ }^{15}$.

Therefore, the main objective of the present study was to describe and analyze the cognitive evaluation of patients with chronic pain compared to control subjects with no pain. Finally, we intended to have non specialized clinicians evaluating patients with chronic pain in general and not a specific group through a brief battery of cognitive tests validated for the Brazilian population, encompassing other cognitive domains rather than only working memory. An additional objective was to assess the influence of the main confounding factors that trigger cognitive disorders in chronic pain patients (such as depressive disorder, anxiety disorder, unrefreshed sleep and the use of medications acting on the central nervous system). To do so, we tried to use statistical methods that could evaluate the influence of different variables on cognition.

\section{METHOD}

A six-month cross-sectional descriptive study was conducted on outpatients with non-oncologic chronic pain. The patients in the chronic pain group (CPG) were recruited in the waiting rooms of the outpatient clinics of various specialties (orthopedics, neurology, internal medicine) by answering the question: "Throughout our lives, most of us have had pain from time to time (such as minor headaches, sprains and toothaches). Have you had pain, other than these everyday kinds of pain, in the last three months?"16. Next, they were interviewed in a specific room at the University Hospital.
Following the criteria of the International Association for the Study of Pain ${ }^{17}$, those with chronic pain of more than three months duration were included. Those who did not report any kind of pain were assigned to the control group (CG) and were paired by sex, age and education.

Inclusion criteria were presence of non-oncologic chronic pain lasting more than 3 months, and age ranging from 18 to 60 years. A patients signed a written informed consent. Exclusion criteria were presence of diagnosed dementia, organic brain dysfunction, illiteracy, and age above 60 years old. The criteria used to exclude dementia were those suggested by DSM $5^{18}$. Through interviews with patients, it was asked if they had memory impairment and others, such as aphasia, apraxia, agnosia or deficit in executive functioning, causing significant functional impairment. The study was approved by the Regional Ethics Committee (CAE 27339914.4.0000.5378).

The patients were divided into age groups of 18 to 30 years, 31 to 40 years, 41 to 50 years, and 51 to 60 years and further divided into different educational level groups of 1 to 4 years of schooling, 5 to 8 years of schooling, 9 to 12 years of schooling, and more than 12 years of schooling.

\section{Data investigated}

1) Demographic data such as age, sex and educational level.

2) A semi-structured questionnaire based on the Brief Pain Inventory validated for the Brazilian population ${ }^{16}$ was applied to the patients with chronic pain. Clinical data regarding acute pain symptoms, location and characteristics of the pain, pain intensity (numeric pain scale), use of analgesics, and affective pain data were obtained.

3) Comorbidities were determined for both groups, such as depressive disorder and anxiety disorder (Diagnostic and Statistical Manual of Mental Disorders criteria were applied to all patients) ${ }^{18}$, sleep aspects ("Do you have any kind of sleep problems such as insomnia, unrefreshed sleep or sleep apnea?"), alcohol abuse ("How many doses of alcohol do you drink weekly?), history of head trauma ("Have you ever had any kind of head trauma?") and other comorbidities that were previously diagnosed by other clinicians such as thyroid dysfunction, diabetes and hypertension.

4) Use of medications, for both groups.

5) Both groups were submitted to neuropsychological evaluation by the Montreal Cognitive Assessment (MoCA) test $^{19,20}$ validated for Portuguese, and Stroop Victoria Test $(\mathrm{ST})^{21}$ (for attention and working memory). We used Verbal Fluency (VF) and Clock Drawing (CD) as part of the MoCA.

MoCA: The Montreal Cognitive Assessment test is a brief screening tool for cognitive impairment. It is a one-page protocol that assesses eight cognitive domains (attention, executive functions, calculations, language, working memory and recall, abstraction, orientation, and visuospatial processing), including a Clock Drawing Test and an oral Verbal Fluency Test $^{21,22}$. 
Stroop Victoria Test: The test assesses selective attention and inhibitory control of interfering stimuli ${ }^{21}$.

\section{Statistical analysis}

Data were analyzed statistically using the Statistical Package for the Social Sciences (SPSS software version 18.0; IBM Corporation) ${ }^{24}$. Categorical variables were analyzed by the chi-square test or Fisher exact test according to the expected frequency in the cells. The level of significance was set at 0.05 . Numeric variables were analyzed by the Kolmogorov-Smirnov test to define their type of distribution, and those with normal distribution were analyzed by parametric tests such as the Student t-test or analysis of variance (ANOVA). Variables with non-normal distribution were analyzed by the nonparametric Mann-Whitney test.

To analyze patients regarding their performance in the MoCA test, we used a linear regression model with MoCA test as the dependent variable, and chronic pain, depressive disorder, anxiety disorder, hypothyroidism, and unrefreshed sleep as the independent variables.

\section{RESULTS}

A total of 100 patients were recruited in the outpatients clinics of different medical specialties. Of these, 45 had chronic pain according to the criteria of the International Association for the Study of Pain, 10 were excluded (5 were illiterate and 5 refused to participate in the study) and 45 of them had no kind of pain and were used as controls.

Among patients assigned to the Chronic Pain Group (CPG) and to the Control Group (CG), 34 (75.5\%) were women. Mean age was 46.9 (Standart deviation - SD 11.9) years for CPG patients and 45.1 (SD 10.4) years for CG subjects. Regarding educational level, in both groups, $23(51.1 \%)$ patients had 8 to 11 years of schooling (High school level) (Table 1).

Among CPG patients, mean pain intensity measured by the Numeric Pain Scale was 7.8 (range: 4-10 points). The mean number of days with pain in a month was 22.4. The most frequent diagnoses were ostheomuscular pain in 21 (23.3\%) patients, fibromyalgia in $7(7.8 \%)$, neuropathic pain in $8(8.9 \%)$, and headache in $5(5.6 \%)$, with $4(4.4 \%)$ patients having no definite diagnosis for their pain.

Table 1. Demographic data of the group of patients with Chronic Pain (CPG) and the Control Group (CG).

\begin{tabular}{lcc}
\hline & CPG & CG \\
\hline Mean age & $46.9($ SD*11.9) & $45.1(S D * 10.4)$ \\
Gender (Female) & $34(75.5 \%)$ & $34(75.5 \%)$ \\
Up to 4 years of schooling & $8(17.7 \%)$ & $8(17.7 \%)$ \\
Schooling 4-8 years & $6(13.3 \%)$ & $6(13.3 \%)$ \\
Schooling 8-11 years & $23(51.1 \%)$ & $23(51.1 \%)$ \\
More than 12 years of schooling & $8(17.7 \%)$ & $8(17.7 \%)$ \\
\hline *SD: standart deviation. & &
\end{tabular}

Dipyrone was the abortive pain medication most frequently used (23-51.1\%), followed by non-steroidal anti-inflammatories (5-11.1\%), opioids (5-11.1\%), and paracetamol (4-8.8\%). Since the control group did not have any kind of pain, they did not use any analgesics.

Antidepressants were used by 8 (17.7\%) CPG subjects and by 8 (17.7\%) CG subjects and benzodiazepines by $3(6.6 \%)$ CPG subjects and 4 (8.8\%) CG subjects. Regarding antidepressants, in the CPG, none patient used tryciclic antidepressants, 1 patient used dual antidepressant (serotonin and noradrenaline reuptake inhibitor) and 7 patients used selective serotonin reuptake inhibitors. In the $\mathrm{CG}$, none patient used tryciclic antidepressants and 8 patients used selective serotonin reuptake inhibitors. When comparing a group of all patients using antidepressants, benzodiazepines or opioids with another group taking no antidepressants, benzodiazepines or opioids, and their performance in the MoCA test, we found that 69 patients using no medication (antidepressants, benzodiazepines or opioids) had a MoCA mean value of 22.59 (Sd 3.7) and 21 patients using medication (antidepressants, benzodiazepines or opioids) had a MoCA mean value of 23.14 (SD 3.7). There was no difference between groups in MoCa test performance ( $p=0.610$, Mann-Whitney test), Verbal Fluency ( $p=0.504$, Mann-Whitney test), Clock Drawing ( $p=0.687$, Mann-Whitney test), Stroop Test 1 ( $p=0.746$, Mann-Whitney test), Stroop Test 2 ( $\mathrm{p}=0.992$, Mann-Whitney test), Stroop Test 3 ( $p=0.746$, Mann-Whitney test).

The most frequent comorbidities detected in the CPG were generalized anxiety disorder (27-60\%), major depressive disorder (18-40\%), unrefreshed sleep (23-51.1\%), hypertension (11-24.4\%), hypothyroidism (8-17.7\%), and diabetes (5-11.1\%). No patient reported previous head trauma or alcohol abuse.

In the $\mathrm{CG}$, the most frequent comorbidities were generalized anxiety disorder (17-37.7\%), major depressive disorder (8-17.7\%), unrefreshed sleep (7-15.5\%), hypertension (6-13.3\%), hypothyroidism (2-4.4\%), and diabetes (5-11.1\%). No subject reported previous head trauma or alcohol abuse.

There was a significant relationship between chronic pain and major depressive disorder, generalized anxiety disorder, hypothyroidism, and unrefreshed sleep, when analyzed by the Fisher exact test, with the level of significance set at 0.05 (Table 2).

Table 2. Comparison of the groups [Chronic Pain (CPG) and Control Group CG)] and their comorbidities.

\begin{tabular}{llcc}
\hline Comorbidities & CPG (\%) & CG (\%) & $\mathrm{p}^{*}$ \\
\hline Major depressive disorder & $18(40)$ & $8(17.7)$ & 0.018 \\
Generalized anxiety disorder & $27(60)$ & $17(37.7)$ & 0.029 \\
Unrefreshed sleep & $23(51.1)$ & $7(15.5)$ & 0.000 \\
Hypertension & $11(24.4)$ & $6(13.3)$ & 0.141 \\
Hypothyroidism & $8(17.7)$ & $2(4.4)$ & 0.045 \\
Diabetes & $5(11.1)$ & $5(11.1)$ & 0.630 \\
\hline *Fisher test. & & &
\end{tabular}


Regarding the cognitive tests applied, the means of MoCA scores were 21.5 (SD 3.8) for CPG and 23.9 (SD 3.1) for CG. As MoCA results are numeric variables with non-normal distribution, we compared groups (CPG and CG) using the nonparametric Mann-Whitney test $(\mathrm{p}=0.002)$. The mean score for Verbal Fluency was 11.9 (SD 3.8) for CPG and 15.4 (SD 5.9) for CG. Since Verbal Fluency results are numeric variables with non-normal distribution, we used the nonparametric Mann-Whitney test $(\mathrm{p}=0.001)$. In the Clock Drawing test, with a 0 to 3 score, the mean score was 1.9 (SD 1.1) for CPG and 2.4 (SD 0.8) for CG ( $\mathrm{p}=0.022$, Mann-Whitney test).

In the Stroop test, the mean time was 22.3 (SD 6.2) seconds for CPG and 14.5 (SD 5.9) for CG ( $p=0.000$, Mann-Whitney test) in the first stage, 29.9 (SD 7.0) for CPG and 21 (SD 8.8) and for CG ( $\mathrm{p}=0.000$, Mann-Whitney test) in the second stage, and 35.5 (SD 9.6) for CPG and 28.3 (SD11.9) for CG ( $p=0.000$, Mann-Whitney test) in the third stage (Table 3).

Although the patients with major depressive disorder and generalized anxiety disorder had a worse performance in the MoCA test ( $p=0.037$ and $p=0.034$, Mann-Whitney test), the presence of chronic pain was an independent factor for this result.

To analyze patients regarding their performance in the MoCA test, we used a linear regression model, with the MoCA as the dependent variable and chronic pain, depressive disorder, anxiety disorder, hypothyroidism, and unrefreshed sleep as independent variables. We detected that the Chronic Pain ( $p=0.015)$ variable was an independent factor for the worse results obtained with the MoCA, but not for depressive disorder $(p=0.279)$, anxiety disorder $(p=0.632)$, hypothyroidism $(p=0.622)$, or unrefreshed Sleep $(\mathrm{p}=0.587)($ Table 4).

Table 3. Neuropsychological tests applied to the group of Patients with Chronic Pain (CPG) and to the Control Group (CG).

\begin{tabular}{lccc}
\hline \multirow{2}{*}{ Test } & \multicolumn{2}{c}{ Mean } & \multirow{2}{*}{ p $^{*}$} \\
\cline { 2 - 3 } & CPG & CG & \\
\hline MoCa & $21.5($ Sd 3.8) & $23.9($ Sd 3.1) & 0.002 \\
MoCa verbal fluency & $11.9($ Sd 3.8) & $15.4($ Sd 5.9) & 0.001 \\
MoCa clock drawing & $1.9($ Sd 1.1) & $2.4($ Sd 0.8) & 0.022 \\
Stroop test (1) & $22.3($ Sd 6.2) & $14.5($ Sd 5.9) & 0.000 \\
Stroop test (2) & $29.9($ Sd 7.0) & $21($ Sd 8.8) & 0.000 \\
\hline Stroop test (3) & $35.5($ Sd 9.6) & $28.3($ Sd 11.9) & 0.000 \\
\hline
\end{tabular}

MoCa: Montreal cognitive assessment; Sd: seconds; *Mann-Whitney test.

\section{DISCUSSION}

We have described and analyzed here the cognitive aspects of patients with chronic pain compared to controls with no pain. Although the number of patients studied here was small, we were able to match controls for sex, age and schooling, and we have assessed the main factors triggering the cognitive disorders. When testing 90 recruited patients in a multidisciplinary medical center with a brief cognitive test battery, patients with chronic pain had worse performance in clinically significant neuropsychological tests than those of the controls.

The results of our study added new knowledge to previous studies, which also found a lower performance in neuropsychological tests for patients with chronic pain. First of all, we evaluated patients with chronic pain in general, not a specific group as fibromyalgia or lumbar pain ${ }^{5,6,7}$. In addiction, we intended to investigate other cognitive domains rather than only working memory ${ }^{12}$, including tests that could be brief and easily administered by non specialized clinicians of a primary care health service. The MoCA is a brief screening test for cognitive dysfunction that assesses eight cognitive domains (attention, executive functions, calculations, language, working memory and recall, abstraction, orientation, and visuospatial processing), representing a sensitive tool for stages of mild cognitive deficit. In this group of chronic pain patients, the scores of the MoCA were lower those of the control subjects, suggesting a mild cognitive deficit for this group of patients.

The analysis of the items of the MoCA demonstrated worse performance for the CPG group in $\mathrm{CD}$ and VF, that are part of the MoCA and widely used in neuropsychological evaluation. CD is supposed to evaluate visual perception functions and VF, language efficiency. However, a deficit in $\mathrm{CD}$ and VF can be related to an executive dysfunction or to an attention deficit because, as many others neuropsychological tests are interactive and depend on more than one ability to reach and an optimal performance.

Interestingly, a deficit on the Stroop test was demonstrated, that evaluates inhibition control related to attention and executive functions. It was observed that the CPG took more time to complete the Stroop test in all of three conditions, indicating a problem of processing mental speed time rather than of cognitive inhibition. This difference in the

Table 4. Linear regression model, where the MoCa test was the dependent variable, controlled for the independent variables chronic pain, depressive disorder, anxiety disorder, hypothyroidism, and unrefreshed sleep.

\begin{tabular}{lcccc}
\hline Model & Unstandardized coefficients B & Std error & $\mathrm{p}^{*}$ & \multicolumn{1}{c}{$95 \% \mathrm{Cl}$} \\
\hline Chronic pain & -2.06 & 0.83 & 0.015 & $-3.71-0.40$ \\
Generalized anxiety disorder & -0.40 & 0.83 & 0.632 & $-2.05-1.25$ \\
Unrefreshed sleep & 0.48 & 0.88 & 0.587 & $-1.27-2.23$ \\
Hypothyroidism & 0.61 & 1.23 & 0.622 & $-1.85-3.07$ \\
Major depressive disorder & -1.02 & 0.93 & 0.279 & $-2.88-0.84$ \\
\hline
\end{tabular}

MoCa: Montreal cognitive assessment; * $p$-value: linear regression model. 
performance of the CPG could be attributed more to an attention dysfunction than to an executive deficit. Thus, probably the global mild cognitive impairment displayed by CPG could be related to an attention disorder that usually interferes with other cognitive domains.

Most studies suggest that systematic assessment in other centers that treat chronic pain patients would be desirable, since there is wide variability between subgroups of patients with pain and confounding variables. We did not detect a previous study in this area including patients in a developing country like Brazil. Once more, the neuropsychological techniques adopted, such as the MoCA test ${ }^{18}$, have been validated for the Brazilian population, with a cut off of 26 , for patients with more than 12 years of schooling. Low MoCA scores for the control group can be probably explained not only by the schooling level of the patients (most of them had up to 11 years of schooling), but by an inefficient educational brazilian system. We have patients that reach 11 years of schooling, with a weak performance in simple tasks, as mathematical calculus and portuguese writing.

We opted to use statistical methods that could evaluate the influence of different variables on cognition. The main variables that might influence the performance of patients with chronic pain in neuropsychological tests have not been analyzed before with specific statistical models. A previous study identified some possible reasons for cognitive complaints, including the presence of depressive symptoms (which occur in 40 to $50 \%$ of patients with chronic pain). Twenty percent of patients with chronic pain scored below the cut-off point in neuropsychological tests ${ }^{15}$. Hae Juin Ko et al. demonstrated that patients with depressive symptoms have more subjective memory complaints, but it is not known for sure whether pain would be an independent factor, since the authors did not use statistical methods that could evaluate its influence ${ }^{25}$. In another previously published study regarding working memory function in persons with chronic pain, confounding factors were not fully analyzed ${ }^{12}$.

Possible detrimental effects of medication are an important issue in the context of chronic pain and cognitive functions. Some studies found that opioids improved cognitive functions. Another study stated that there were no differences in neuropsychological functioning between patients receiving only opioids and a no-pain-medication group $^{15}$. In our study, we found a low use of medications interfering with the central nervous system, with the type and quantity of medications being similar for the CPG and CG. Neuropsychological functioning was similar for the pain-medication and no-pain-medication groups.

Finally, people with sleep problems often have cognitive impairment ${ }^{26}$. Probably, a sleep disruption may lead to a deterioration of cognition because memory consolidation is performed during the sleep time. Although in our study most of chronic pain patients reported unrefreshed sleep (23-51.1\%), this comorbidity was not influencing the performance in MoCa test, as analyzed by a linear regression model ( $\mathrm{p}=0.587)$.

The present study has some methodological limitations that are worth considering. First, the number of patients studied here was small. Secondly, a brief cognitive assessment was selected to be applied by a non specialized clinician in a health primary care service. Probably, the neuropsychological assessment should be expanded to other tests that could clarify the questions about the cognition of chronic pain aroused by this study. However, the present results could be an initial step towards the development of a brief cognitive battery of tests that could be applied to a health primary care service.

In conclusion, the relationship between chronic pain and cognitive deficits represents a genuine reality not to be neglected in the daily routine of physicians who work with pain management. In addition, these deficits include not only impairment of working memory, but also a worse performance in a brief screening test for cognitive impairment like the MoCA test, and others tests as Verbal Fluency Test, Clock Drawing Test and Stroop Test. The main confounding factors that trigger cognitive disorders in chronic pain patients were assessed and it was observed that chronic pain is an independent factor for this. The comprehension of its causes deserves further studies for clarification.

\section{References}

1. Teixeira MJ. Dor: manual para o clínico. São Paulo: Atheneu, 2006

2. Warfield CA, Bajwa ZH. Principles and practice of pain medicine. $2 n d$ ed. New York: McGraw-Hill; 2004.

3. Merskey H, Bogduk N. Classification of chronic pain: description of chronic pain syndromes and definition of pain terms. Seattle: IASP Press; 1994.

4. Andersson HI, Ejlertsson G, Leden I, Rosenberg C. Chronic pain in a geographically defined general population: studies of differences in age, gender, social class, and pain localization. Clin J Pain. 1993;9(3):174-82. doi:10.1097/00002508-199309000-00004
5. Glass JM, Park DC, Minear M, Crofford LJ. Memory beliefs and function in fibromyalgia patients. J Psychosom Res. 2005;58(3):263-9. doi:10.1016/j.jpsychores.2004.09.004

6. Schiltenwolf M, Akbar M, Hug A, Pfüller U, Gantz S, Neubauer E et al. Evidence of specific cognitive deficits in patients with chronic low back pain under long-term substitution treatment of opioids. Pain Physician. 2014;17(1):9-20.

7. Grace GM, Nielson WR, Hopkins M, Berg MA. Concentration and memory deficits in patients with fibromyalgia syndrome. J Clin Exp Neuropsychol. 1999;21(4):477-87. doi:10.1076/jcen.21.4.477.876 
8. Apkarian V, Hashmi JA, Baliki MN. Pain and the brain: specificity and plasticity of the brain in clinical chronic pain. Pain. 2011;152(3 suppl):S49-64. doi:10.1016/j.pain.2010.11.010

9. Legrain V, Damme SV, Eccleston C, Davis KD, Seminowicz DA, Crombez G. A neurocognitive model of attention to pain: behavioral and neuroimaging evidence. Pain. 2009;144(3):230-2. doi:10.1016/j.pain.2009.03.020

10. Rohling ML, Green P, Allen LM 3rd, Iverson GL. Depressive symptoms and neurocognitive test scores in patients passing symptom validity tests. Arch Clin Neuropsychol. 2002;17(3):205-22. doi:10.1093/arclin/17.3.205

11. Suhr JA. Neuropsychological impairment in fibromyalgia: relation to depression, fatigue, and pain. J Psychosom Res. 2003;55(4):321-9. doi:10.1016/S0022-3999(02)00628-1

12. Berryman C, Stanton TR, Bowering KJ, Tabor A, McFarlane A, Moseley GL. Do people with chronic pain have impaired executive function? A meta-analytical review. Clin Psychol Rev. 2014;34(7):563-79. doi:10.1016/j.cpr.2014.08.003

13. Liu X, Li L, Tang F, Wu S, Hu Y. Memory impairment in chronic pain patients and the related neuropsychological mechanisms: a review. Acta Neuropsychiatr, 2014;26(4):195-201. doi:10.1017/neu.2013.47

14. Berryman C, Stanton TR, Bowering JK, Abby T, McFarlane, A, Moseley, LG. Evidence for working memory deficits in chronic pain: A systematic review and meta-analysis. Pain. 2013;154(8):1181-96. doi:10.1016/j.pain.2013.03.002

15. Wolrich J, Poots AJ, Kuehler BM, Rice AS, Rahman A, Bantel C. Is number sense impaired in chronic pain patients? Br J Anaesth. 2014;113(6):1024-31. doi:10.1093/bja/aeu255

16. Roth RS, Geisser ME, Theisen-Goodvich M, Dixon PJ. Cognitive complaints are associated with depression, fatigue, female sex, and pain catastrophizing in patients with chronic pain. Arch Phys Med Rehabil. 2005;86(6):1147-54. doi:10.1016/j.apmr.2004.10.041

17. Landrø NI, Fors EA, Våpenstad LL, Holthe $\emptyset$, Stiles TC, Borchgrevink PC. The extent of neurocognitive dysfunction in a multidisciplinary pain centre population. Is there a relation between reported and tested neuropsychological functioning? Pain. 2013;154(7):972-7. doi:10.1016/j.pain.2013.01.013

18. Toledo FO, Barros PS, Herdman M, Vilagut G, Reis GC, Alonso $\mathrm{J}$ et al. Cross-cultural adaptation and validation of the Brazilian version of the Wisconsin Brief Pain Questionnaire.J Pain Symptom Manage. 2013;46(1):121-30. doi:10.1016/j.jpainsymman.2012.07.017

19. Treede RD, Rief W, Barke A, Aziz Q, Bennett MI, Benoliel R et al. A classification of chronic pain for ICD-11. Pain. 2015;156(6):1003-7. doi:10.1097/j.pain.0000000000000160

20. American Psychiatry Association. Diagnostic and statistical manual of mental disorders - DSM - 5. 5th ed. Washington: American Psychiatric Association; 2013.

21. Bertolucci PHF, Sarmento ALR, Wajman JR. Brazilian Portuguese version for the Montreal Cognitive Assessment (MoCA) and the preliminary results. Alzheimers Dement. 2008;4(4 Suppl):686. doi:10.1016/j.jalz.2008.05.2127

22. Freitas S, Simões MR, Martins C, Vilar M, Santana I. Estudos de adaptação do Montreal Cognitive Assessment (Moca) para a população portuguesa. Aval Psicol. 2010;9(3):345-57.

23. Stroop R. Studies of interference in serial verbal reactions.J Exp Psychol. 1935;18(6):643-62. doi:10.1037/h0054651

24. Mitrushina MM, Boone KB, D’Elia LF. Handbook of normative data for neuropsychological assessment. New York: Oxford University Press; 1999.

25. Agrell B, Dehljn O. The clock-drawing test. Age Ageing. 1998;27(3):399-403. doi:10.1093/ageing/27.3.399

26. SAS/ STAT User's Guide, Version 8. Cary, NC: SAS Institute Inc., 1999

27. Ko HJ, Seo SJ, Youn CH, Kim HM, Chung SE. The association between pain and depression, anxiety, and cognitive function among advanced cancer patients in the hospice ward. Korean J Fam Med. 2013;34(5):347-56. doi:10.4082/kjfm.2013.34.5.347

28. Silva RAPC. Sleep disturbances and mild cognitive impairment: a review. Sleep Science. 2015;8(1):36-41. doi:10.1016/j.slsci.2015.02.001 